\title{
Misperception in sentences but not in words: Speech perception and the phonological buffer
}

\author{
Charlotte Jacquemot \\ INSERM U421 Equipe Neuropsychologie Interventionnelle, Créteil, and ENS, Paris, France; \\ Institute of Cognitive Neuroscience, University College London, London, UK \\ Emmanuel Dupoux \\ Laboratoire de Sciences Cognitives et Psycholinguistique, EHESS-ENS-CNRS, Paris, France \\ Odile Decouche \\ INSERM U421 Equipe Neuropsychologie Interventionnelle, Créteil, and ENS, Paris, France
}

Anne-Catherine Bachoud-Lévi

INSERM U421 Equipe Neuropsychologie Interventionnelle, Créteil, ENS, Paris, France;

Unité de Neuropsychologie, Service de Neurologie, Hôpital Henri Mondor, Créteil, France

\begin{abstract}
We report two case studies of aphasic patients with a working-memory impairment due to reduced storage in the phonological buffer. The two patients display excellent performance in phonological discrimination tasks as long as the tasks do not involve a memory load. We then show that their performance drops when they have to maintain fine-grained phonological information for sentence comprehension: They are impaired at mispronunciation detection and at comprehending sentences involving minimal word pairs. We argue that the phonological buffer plays a role in sentence perception during the phonological analysis of the speech stream: It sustains the temporary storage of phonological input in order to check and resolve phonological ambiguities, and it also allows reexamination of the phonological input if necessary.
\end{abstract}

\section{INTRODUCTION}

When we hear a sentence, a number of different processing levels are involved at the same time: acoustic, phonological, morpho-lexical, syntactic, semantic pragmatic, and so on. The incoming signal is processed in a gradual and incremental way and cascades through these processing levels (Gaskell \& Marslen-Wilson, 1996, 1998; McClelland \& Elman, 1986). At each level,

Correspondence should be addressed to Charlotte Jacquemot, INSERM U421, Equipe Neuropsychologie Interventionnelle, Université Paris XII, 8, rue du Général Sarrail, 94010 Créteil cedex, France (Email: charlotte.jacquemot@ens.fr).

We thank Anne Christophe, Isabelle Darcy, Lisa Feigenson, and Gayaneh Szenkovits for their help with this work, and Sophie Scott, Narly Golestani, and Jonas Obleser for proofreading the article. We are also grateful to Randi Martin and two anonymous reviewers for their many helpful comments on earlier versions of this paper. This work was supported by ESRC, the Fyssen Foundation, and the INSERM through an Interface grant, and an Avenir grant. 
however, the analysis of the current chunk of signal has to be integrated with a temporary representation of the immediate past. Hence, each processing level uses its own memory resource or buffer-for example, acoustic buffer, phonological buffer, morpho-lexical buffer, syntactic buffer, and so on. The link between these memory resources and the more classical notions of verbal working memory has still to be specified in full detail. In the classical view, verbal working memory is composed of a phonological buffer, in which auditory material is registered, and an active subvocal rehearsal component, which refreshes and maintains the information in the phonological buffer (Baddeley, 1986). More recently it has been suggested that verbal working memory consists of the collection of memory resources that are used during sentence comprehension (Martin \& Romani, 1994; Martin, Shelton, \& Yaffee, 1994). This raises the prediction that deficits in verbal working memory should always be associated with some impairment in sentence processing. However, one could propose that sentence processing will not always be impaired in the same way depending on the type of buffer that is damaged. In this paper, we specifically address the role of the phonological buffer in sentence processing.

Most research has focused on the possibility that verbal working memory is crucial to sentence comprehension with respect to the syntactic and semantic processing of long and complex sentences (Vallar \& Baddeley, 1984a). Support for these assumptions comes from neuropsychological patients with very impaired memory spans who display difficulties with sentence comprehension (Caramazza, Berndt, Basili, \& Koller, 1981; Martin \& Feher, 1990; Saffran \& Marin, 1975; Saffran \& Martin, 1990). However, recent studies suggest that verbal working memory is not always involved in syntactic and semantic processing: Some patients with severely reduced verbal span have shown preserved sentence comprehension skills and normal processing of syntactically complex sentences (Butterworth, Campbell, \& Howard, 1986; Caplan \& Waters, 1990, 1999; Hanten \& Martin, 2001; Martin, 1987, 1990,
1993; Van der Linden \& Poncelet, 1998; Waters, Caplan, \& Hildebrandt, 1991). Such a finding can be partly explained by the fact that an impairment in verbal working memory may be due to several different causes and that only some of these result in syntactic or semantic deficits.

In support of this idea, first, there is some evidence that in the verbal span tasks that are traditionally used to test working memory, lexical and semantic variables play a role in performance (Cowan, 2001; Martin \& Saffran, 1997). Several studies have demonstrated, for example, that for normal subjects word span is greater than pseudoword span (Cowan, 2001; Crowder, 1978; Hulme, Maughan, \& Brown, 1991). This effect occurs because words have semantic representation in addition to phonological representation whereas pseudowords have only phonological representation. The separate contribution of phonological and semantic information in span tasks has been reported by Martin et al. (1994) who described patients having a disruption of one but not the other component. They investigated two patients with reduced memory spans. One of the patients, E.A., showed worse retention in span tasks using phonological information (pseudowords) than in span tasks using semantic information (words). The other patient, A.B., showed the reverse pattern. Moreover, E.A. was more impaired than A.B. on sentence repetition, which draws on phonological retention to ensure verbatim retention, whereas A.B. was more impaired than E.A. on sentence comprehension, which necessarily requires semantic retention. The dissociation of semantic from phonological information in these patients suggests separable semantic and phonological components in verbal working memory as well as a separate role of these components in sentence processing (Hanten \& Martin, 2001; Martin \& He, 2004; Martin, Lesch, \& Bartha, 1999b; Martin \& Romani 1994).

Second, even if one looks at deficits restricted to phonological memory components, one might still expect different patterns of associations between aspects of sentence processing and memory abilities. For instance, phonological buffer is involved 
in holding temporary information whereas subvocal rehearsal is presumably not used during sentence processing. If so, one could expect to observe few consequences of a verbal workingmemory impairment that is due to a selectively subvocal rehearsal deficit in sentence processing (see Martin, 1990).

In this paper, we hypothesize that the phonological buffer plays a role in sentence processing, specifically in the phonological analysis of sentences. We propose that the phonological buffer is involved in situations where accessing and storing of several adjacent syllables are required. Such a situation arises during word segmentation in which the detailed phonetic and prosodic shapes of syllables are used to extract probable word boundaries (McClelland \& Elman, 1986; Norris, 1994). As phonetic information is derived from the incoming auditory signal, it is necessary that the sequence of phonemes be maintained until lexical access is achieved. Another such situation arises during the disambiguation of mispronunciation or during misperception. In both cases, the processing system may have to check the current lexical hypothesis with the phonological representation of the word in order to resolve the error or ambiguity. Therefore we propose that the phonological buffer is involved in tasks in which the storage of several syllables is required. According to this, one possible role of the buffer is a prelexical component necessary for maintaining phonological input while word segmentation is processed. A second role is to serve as a backup system when a disambiguation of mispronunciation or of a misperception is required. Here, we report the study of two patients with phonological buffer deficits, and we explore its impact on tasks such as word segmentation, mispronunciation detection, or phonological ambiguity resolving, involving the storage of several syllables. We predict that the patients will be impaired on such tasks.

This paper is divided in three parts. In the first part, we evaluate the verbal working-memory performance of the two patients with a series of experiments focusing on phonological memory components, on the phonological buffer, and on subvocal rehearsal. In the second part, we evaluate phonological processing performance in isolation with tasks involving only one or two items. In the third part, we evaluate patients' performance on sentences and demonstrate that connected speech perception is impaired despite flawless performance on isolated word perception.

\section{CASE REPORTS}

Patient F.L. was a right-handed 59-year-old computer specialist. Five years before participating in this study, he had a stroke leading to global aphasia. A computed tomography (CT) scan (at admission) and a magnetic resonance imaging (MRI) scan (4 years later) confirmed a left perisylvian stroke. Patient A.T. was a right-handed 55year-old retired human resources consultant. Eight years before participating in this study, he had a stroke leading to global aphasia. A CT scan (at admission) confirmed a left fronto-parietal lesion.

Both patients were tested in 2002 at the neurology department of Henri Mondor Hospital (Créteil, France) in external consultation. They were tested twice a month during 6 months. Both patients had spectacularly improved their speech-processing performance relative to immediately after the stroke. They were fluent in speech production, to the extent that uninformed listeners did not detect abnormalities in their speech. A naming task confirmed their excellent performance in speech production. Picture names were composed of 20 monosyllabic, 20 bisyllabic, and 20 trisyllabic words (for each length, half of the words were of low frequency, and half were of high frequency), 10 quadrisyllabic words (low frequency), and 10 digits. Frequency was matched across the groups. A.T. performed at a 94\% correct level and F.L. at a 95\% correct level suggesting no production deficit. Both patients made only semantic errors (for instance skirt for dress).

The Boston Diagnostic Aphasia Examination (BDAE; see Appendix A and Appendix B) revealed good performance in nearly every test, 
with the exception of comprehension tasks (command A.T.: 10/15) and sentence repetition (F.L.: 7/16) in which both patients made phonological paraphasia and nonresponse errors. Their repetition of words, however, was good (both 8/ 10 , errors were phonemic paraphasias), as was their word discrimination (A.T.: 70/72 and F.L. 71/72).

Both patients complained about a deficit in word perception, especially in the presence of noise or of other conversations. There were members of the same aphasic society and demonstrated a strong motivation to understand their speech perception difficulties. This perceptual problem was the primary reason for their coming to the neurology department. Interestingly, both patients independently reported always confusing the names of two people that they mutually knew (namely Mr Perge and Mr Berge), which differed only in the voicing of the first phoneme.

\section{VERBAL WORKING MEMORY}

We first assessed verbal working memory of the patients with a standard digit span task, which globally evaluates verbal working memory, and then proceeded with more detailed tests aimed at evaluating the phonological buffer and the subvocal rehearsal components (Baddeley, 1986). Some aspects of this model involving a phonological buffer, a rehearsal component, and their associated effects remains controversial (Brown \& Hulme, 1995; Hulme, Surprenant, Bireta, Stuart, \& Neath, 2004; Jones, Macken, \& Nicholls, 2004; Nairne, 2002) but its structure is still widely accepted (Baddeley, Chincotta, Stafford, \& Turk, 2002; Cowan, Baddeley, Elliott, \& Norris, 2003; Mueller, Seymour, Kieras, \& Meyer, 2003), and we therefore used it as a framework for our study. Following standard procedures, we tested for the integrity of subvocal rehearsal by evaluating word length effect and primacy effect. To test the phonological buffer, we evaluated the recency effect and the phonological similarity effect using pseudowords.

\section{2}

COGNITIVE NEUROPSYCHOLOGY, 2006, 23 (6)

\section{Digit span}

In the standard digit span task, the patients had to repeat arbitrary sequences of digits. The digit sequences were presented at a rate of one digit per second, starting with sequences of size 2. When the response was correct, the sequence length was increased by one item. Forward span (serial recall of items in forward order) and backward span (serial recall of items in reverse order) were tested twice.

A.T. had a forward span of 5 items and a backward span of 4 items, which is within normal limits. F.L. had a forward span of 3 and a backward span of 3 , which is below normal performance.

\section{Word length effect}

Performance on verbal working-memory tasks is influenced by the length of the stimuli to be remembered: Span is lower for long words than for short words. The word length effect is assumed to occur because long words take longer to rehearse, increasing the opportunity for the memory trace to decay in the phonological buffer (Baddeley, 1992). This effect of word length is abolished when subvocal rehearsal is prevented by a concurrent articulatory suppression task (Baddeley, Lewis, \& Vallar, 1984; Belleville, Peretz, \& Arguin, 1992; Cowan, 2001; Vallar \& Baddeley, 1984a). Hence the presence of a length effect can be taken as evidence that the subvocal rehearsal component is present (Baddeley, 1986; but see Romani, McAlpine, Olson, Tsouknida, \& Martin, 2005). Here, as in the standard span task, patients were required to listen to a list of words and to repeat the items in the correct order. Words were presented at the rate of one per second. Two sets of stimuli, one composed of 10 monosyllabic words, and another of 10 quadrisyllabic words, were used. The words in the two sets were matched for frequency. Ten trials of increasing sequence length (sequences of two, three, four, five, and six words) were generated at random from each set and were presented in ascending order. No word was presented twice within a sequence. If the patient's repetition of the first three trials of a given sequence length 
Table 1. Word length effect: Number of correct responses according to word length and sequence length

\begin{tabular}{|c|c|c|c|c|c|c|}
\hline \multirow{2}{*}{$\begin{array}{l}\text { Sequence } \\
\text { length }^{\mathrm{a}}\end{array}$} & \multicolumn{2}{|c|}{ A.T. } & \multicolumn{2}{|c|}{ F.L. } & \multicolumn{2}{|c|}{ Control subjects } \\
\hline & Mono & Quadri & Mono & Quadri & Mono & Quadri \\
\hline 2 & 10 & 7 & 10 & 10 & $10( \pm 0)$ & $10( \pm 0)$ \\
\hline 3 & 10 & 3 & 10 & 4 & $10( \pm 0)$ & $10( \pm 0)$ \\
\hline 4 & 3 & 0 & 2 & 0 & $10( \pm 0)$ & $7.8( \pm 2)$ \\
\hline 5 & & & & & $7.2( \pm 2.5)$ & $2.8( \pm 2.7)$ \\
\hline 6 & & & & & $4.5( \pm 3.7)$ & $0.9( \pm 1.4)$ \\
\hline
\end{tabular}

Note: Mono $=$ monosyllabic words. Quadri $=$ quadrisyllabic words. Standard deviations in parentheses.

${ }^{\mathrm{a}} N=10$ for all sequence lengths.

was correct, we assumed that this length was within his span and proceeded to the following string length (Vallar \& Baddeley, 1984a). If the patient's repetition of all 10 trials of a given sequence length was incorrect, we did not carry on with the ascending sequence length and stopped the test.

Results are shown in Table 1 . Results were analysed by estimating the critical sequence length that yielded $50 \%$ of correct recall for each condition (monosyllabic and four-syllable words). We call this sequence length the estimated memory $\operatorname{span}^{1}$ (see Figure 1). Patients' data were compared to data of 10 control subjects (mean age 51.6 years, range $50-55$, mean years of education 13 , range 7-22) analysed in the same way. A.T.'s and F.L.'s spans are 3.92 and 3.86, respectively for the monosyllabic words, and 2.44 and 2.97 for the quadrisyllabic words. The estimated spans of the control subjects (5.66 for the monosyllables words and 4.65 for the quadrisyllabic words), were used to derive $Z$-scores for the patients. Results show that the patients' span is globally impaired in both word conditions (A.T.: Z-score for monosyllabic words $=-3.6, p<.001, Z$-score for quadrisyllabic words $=-3.8, p<.001$; F.L.: $Z$-score for monosyllabic words $=-3.8, p<.001, Z$-score for quadrisyllabic words $=-2.9, p<.001)$. Nevertheless, both A.T. and F.L. show the typical word length

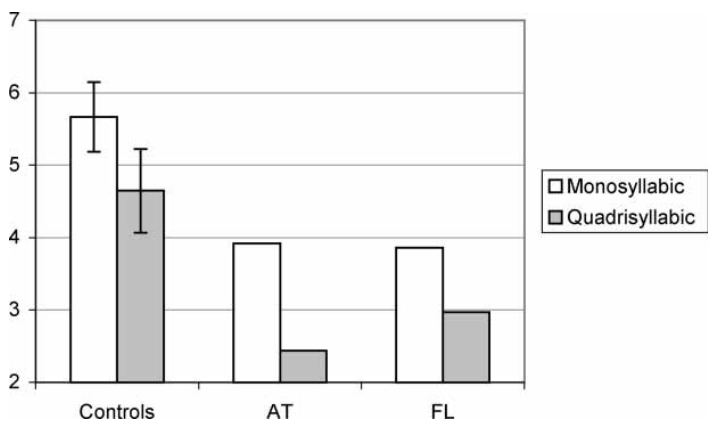

Figure 1. Word length effect. Sequence length yielding 50\% of correct recall for the monosyllabic (in white) and quadrisyllabic words (in grey) conditions.

effect: A.T., $\chi^{2}(1)=9.7, \quad p=.002$, and F.L., $\chi^{2}(1)=34$, as observed in controls, $F(1,98)=$ $8.1, p=.005$, suggesting that their subvocal rehearsal component is intact.

\section{Recency and primacy effects}

Another way to independently evaluate the two components of phonological short-term memory is to study the effect of position in serial recall. The recency effect refers to the fact that the final item in a list is better recalled than items in the middle. It is assumed to reflect the characteristic of the phonological buffer that fades gradually over time; hence a recent item is better represented than an older one. The primacy effect refers to the fact that the first item in a list is better recalled than items in the middle, and, in contrast, it is assumed to reflect the operation of subvocal rehearsal. Indeed, subvocal rehearsal takes time to perform, and one only has the opportunity to use it with the very first items presented (Vallar \& Papagno, 1986).

We assessed the recency and primacy effects using the behavioural data from the monosyllabic word serial recall task described above (word length effect section). We selected data of sequence length of 4 for the patients and 5 for the control subjects since this was a sequence length that was

\footnotetext{
${ }^{1}$ This estimated span was computed by regressing the average recall rate across sequence length with a nonlinear sigmoid function. This was done in SPSS by fitting the data of each individual participant using the nonlinear model.
} 


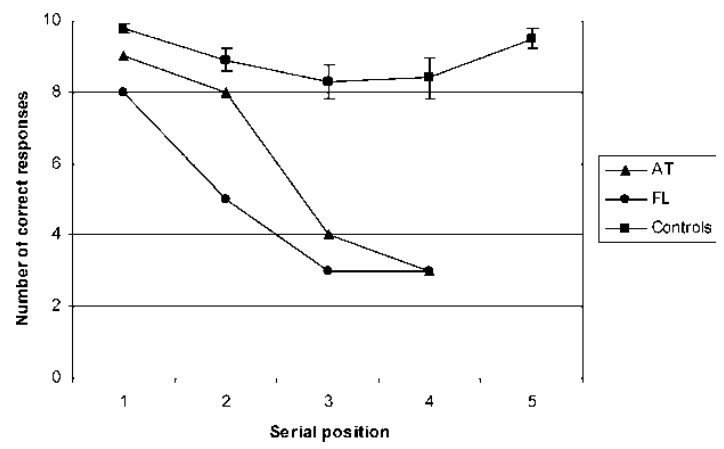

Figure 2. Serial position of correct recall. Number of correct responses $(N=10)$ according to the serial position. Standard error for each serial position is reported for control subjects.

expected to result in an intermediate number of errors, thereby avoiding the possibility of a ceiling or floor effect.

As shown in Figure 2, the patients' ability to recall words in the correct serial position greatly decreases from initial to final position. No advantage for the final position is observed, but there is a clear advantage for the first position (primacy effect). This suggests that subvocal rehearsal is operating in both patients, but that there might be an impairment of the phonological buffer. Results in control subjects show the expected primacy and recency effect: $98 \%$ correct in Position 1, 83\% correct in Position 3, and 95\% correct in Position 5. The first item and the last item are significantly better recalled than the items in the middle position: Position 1 vs. Position 3, $F(1,18)=9.3, p=.007$; Position 3 vs. Position $5, F(1,18)=4.9, p=.041$.

\section{Pseudoword repetition}

Digit span does not provide a very pure measure of phonological short-term memory because of the use of words (digits), in which lexical-semantic information may assist short-term memory storage (Cowan, 2001; Martin et al., 1994). Thus, in order to discard any effect of lexicalsemantic factors on storage capacity, we tested immediate repetition with pseudowords, first with monosyllabic items $(N=16)$ and then with polysyllabic items $(3,5$, or 7 syllables, $N=25)$.

Both patients correctly repeat $96 \%$ of the monosyllabic pseudowords. For the polysyllabic items, their performance is very low: A.T. repeats only correctly $24 \%$ of the polysyllabic items and F.L. 20\%, and their few correct responses are all for trisyllabic items. A.T. and F.L. are impaired relative to control subjects in repeating polysyllabic pseudowords (controls: $87 \%$ correct; A.T.: $Z$-score $=-7.5, p<.001$, and F.L.: $Z$-score $=-7.9, p<.001)$. The very poor performance on polysyllabic pseudowords suggests that the patients are impaired at temporarily holding verbal content in phonological shortterm memory.

\section{Phonological similarity effect}

We explored phonological buffer capacities in more detail. This component of phonological working memory is involved in storing the phonological representations of speech sounds. Serial recall is better when the stimuli to be remembered are phonologically dissimilar rather than similar. This phonological similarity effect is not affected by articulatory suppression, suggesting that it arises in the phonological buffer and not in subvocal rehearsal (Conrad, 1963, 1964; Vallar \& Baddeley, 1984a).

In order to investigate the similarity effect, we constructed a serial recall task involving pseudowords to avoid any lexical-semantic interference. Patients were taught to associate two auditory $\mathrm{CV}-\mathrm{CV}$ pseudowords (C, consonant; V, vowel) to keys "1" and "2", respectively, on a computer keyboard. During a prior training session, patients were asked to choose the correct key according to each of the pseudowords presented in isolation. After training, the patients were presented with progressively longer and more random sequences containing the two items, which they were required to recall and transcribe as sequences of "1" and "2". Six trials of two, three, four, five, or six items were presented in ascending order. In order to minimize subvocal rehearsal of the auditory items, the stimuli were time compressed and were separated by a very short interval 
(Peperkamp \& Dupoux, 2002). Moreover, in order to prevent participants from using a lowlevel echoic memory buffer, every sequence was followed by the word "OK" (Morton, Crowder, \& Prussin, 1971; Morton, Marcus, \& Ottley, 1981). Finally, some phonetic variability was present since each pseudoword was instantiated by one of six acoustically different tokens. The experiment was divided into two parts. In the first part, the two pseudowords were phonologically dissimilar-/rapi/ versus / foga/-while in the second part they were phonologically similar-/mipa/ versus / miba/—and constituted a minimal pair differing in only one distinctive phonetic feature (i.e., voicing). The patients were tested twice.

As previously, results were analysed by estimating the critical sequence length that yielded $50 \%$ of correct recall for each type of contrast (similar or dissimilar). As shown on Figure 3, the control subjects have a span of about 1 item shorter for similar than for dissimilar sequences (4.64 and 5.65, respectively). A.T., in contrast, has a disproportionate difference in span between similar and dissimilar sequences (3.08 and 5.41, respectively, 2.33 items shorter for similar sequence, $Z$-score $=-4.11$, $p<.001)$. This difference is only due to significantly low span for similar sequences $(Z$-score $=-2.6$, $p<.02)$ : The dissimilar sequences yielded a normal span $(Z$-score $=-0.43, p>.1)$. The difference in span between similar and dissimilar

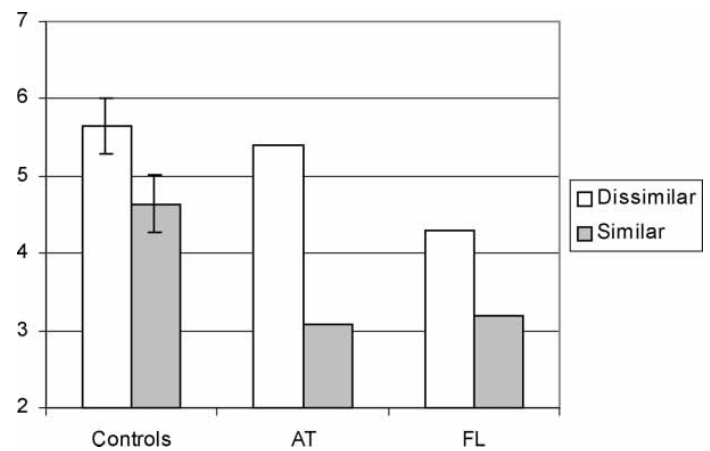

Figure 3. Phonological similarity effect. Sequence length yielding $50 \%$ of correct recall in the dissimilar (in white) and similar (in grey) conditions. sequences for F.L. is within normal limits (3.2 and 4.3, respectively, 1.10 items shorter for similar sequences, $Z$-score $=-0.28, \quad p>.1)$, but his performance is impaired overall for similar as well as dissimilar sequences $(Z$-score $=-2.4, p<.03$; $Z$-score $=-2.4, p<.03$, respectively). The results confirm that A.T. and F.L. have an impaired phonological buffer.

\section{Discussion}

Our exploration of the two patients' performance on several verbal working-memory tasks shows weak serial recall when they were tested using pseudowords, especially for phonologically similar sequences, and the absence of a recency effect. These results suggest that A.T. and F.L. have an impaired phonological buffer. In order to test the phonological buffer capacity specifically and to avoid any influence of lexical-semantic variables, we used a recall task involving phonological similar and dissimilar pseudowords. In this task, we found that patient F.L. has a global reduction in his phonological buffer capacity, whereas A.T. is only impaired for phonologically similar sequences (Figure 3). This observation motivates us to propose that their impairments may have different characteristics. A.T. has a withinnormal memory capacity when it comes to dissimilar sequences (which is consistent with his withinnormal digit span), but has a very large impairment for similar sequences. This disproportionate difficulty with similar sequences suggests that his phonological buffer holds more noisy phonological representations or is subject to abnormal levels of interference from preceding or successive phonological segments (Nairne, 1990). In contrast, F.L. has a normal effect of similarity, but has a more global reduction of verbal working-memory capacity (of about 1.5 items), irrespective of similarity. This suggests a lower capacity for storage in the phonological buffer than normal or more rapid decay of phonological traces.

In contrast, the results of both patients reveal an intact subvocal rehearsal component. This conclusion is supported by the existence of the word length effect in serial recall in both patients.

COGNITIVE NEUROPSYCHOLOGY, 2006, 23 (6)

955 
Even if their performance with short and long words is globally impaired relative to normal controls, they are better at recalling short words than long words (Figure 1). As we have seen above that A.T.'s performance was within normal limits for dissimilar sequences, we could have expected normal performance in the word length effect task in which the stimuli were dissimilar words. This apparent disagreement could be explained when looking at the nature of the errors made by the two patients. The word length effect was assessed with sequences of words from either a list of 10 short words or a list of 10 long words. Similarly to control subjects, F.L. made omission errors and substitution errors in which a given word was substituted by another one from the testing list. In contrast, A.T. made omission errors and substitution errors in which a given word was substituted by another that was phonologically similar to the target but not coming from the testing list (for example dime /dim/ was remembered as dame /dam/, port/por/ as fort /for/, organiser /organize/ as emmagasiner/ãmagazine/). This data supports our hypothesis that proposes that A.T.'s buffer is more susceptible to interference than an intact buffer and could account for A.T.'s errors in the word length effect task.

\section{WORD PROCESSING}

It is currently acknowledged that word comprehension involves several steps (see Figure 4; Franklin, 1989). The first one-phonological decoding - is defined as the translation of acoustic information into discrete segmental categories that belong to the language-that is, the phonological input. Then, lexical recognition itself decomposes into two components: word form selection and lexical retrieval. Word form selection consists in comparing the series of speech sounds of the phonological input to those stored in lexical entries and in selecting the best matched word. Lexical retrieval results from the recovery of syntactic and semantic information attached to the selected lexical entry. In order to investigate the patients' word-processing performance, we constructed

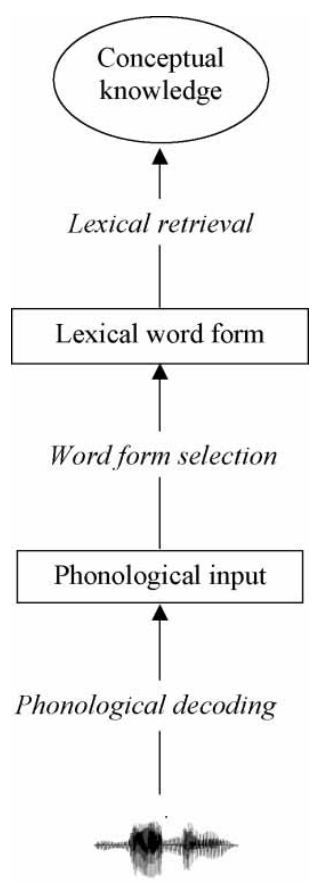

Figure 4. Model of speech perception.

several tasks designed to selectively tap the three steps of speech perception (phonological decoding, word form selection, and lexical retrieval).

\section{Phoneme discrimination}

A deficit in phonological decoding should result in imprecise segmental representations. This should create difficulties in discriminating items that differ minimally in their segmental contents. A.T. and F.L. were tested with an AX discrimination task. A trial was composed of two stimuli (A and $\mathrm{X}$ ) played in succession and separated by $500 \mathrm{~ms}$ of silence. The $\mathrm{X}$ stimulus was either identical to the A (AA trial), or not (AB trial). Sixty pairs of $\mathrm{CV}-\mathrm{CVC}$ bisyllables were constructed. They were minimal pairs, and they differed in a single distinctive phonetic feature on one phoneme (voicing, place, or manner of articulation). This difference could be on the first, second, or third consonant. Half of the pairs were words, and half were pseudowords. The mean word frequency was low (4.68 per million 
on the Brulex Database (Content, Mousty, \& Radeau, 1990)). The pseudowords were obtained by exchanging the first syllables of the words in the list. All the pseudowords were hence phonotactically legal and, moreover, contained the same syllables as the words. These pairs were recorded by two speakers (male and female) and were used to construct $120 \mathrm{AX}$ trials. On half of the trials, the two stimuli were produced by a single speaker (male or female), whereas on the other half, the two stimuli were produced by two different speakers, thus preventing patients from using an acoustic strategy to perform the discrimination task (Jacquemot, Dupoux, Pallier, \& Bachoud-Lévi, 2002). The patients were asked to press a button when the stimuli were the same and another when they were different, irrespective of the speaker's voice.

Both patients performed accurately, and their results did not differ from that of the control subjects (Table 2; A.T.: $Z$-score $=-0.4, p=.4$, and F.L.: $Z$-score $=0.2, \quad p=.4)$. There were no effects of lexical status, location of the change within the item, and nature of the change (voicing, place, or manner of articulation; $p>.1$ ). We also analysed performance according to the number of speakers within trials. Results are

Table 2. Percentage of correct responses in the speech perception tasks

\begin{tabular}{lrrc}
\hline & A.T. & F.L. & Control subjects \\
\hline Phoneme discrimination & 95 & 98 & $96( \pm 4.03)$ \\
$\quad$ With mask & 96 & 96 & \\
$\quad$ With concurrent letter & 94 & 96 & \\
$\quad$ detection task & & & \\
Lexical decision & 96 & 96 & $97( \pm 1.8)$ \\
$\begin{array}{l}\text { Auditory word-picture } \\
\quad \text { matching }\end{array}$ & 99 & 100 & $100( \pm .6)$ \\
$\quad$ Word mispronunciation & 80 & 75 & $96( \pm 4.4)$ \\
$\quad$ detection in sentence & & & \\
$\quad$ Word detection in sentence & 52 & 72 & $92( \pm 5.22)$ \\
$\quad$ In slowed sentence & 58 & 80 & \\
Word segmentation in & 69 & 88 & $97( \pm 3.8)$ \\
$\quad$ ambiguous sentence & & & \\
Word recognition in sentence & 68 & 79 & $93( \pm 4.4)$ \\
\hline
\end{tabular}

Note: Abnormal performance indicated by italics. Standard deviations in parentheses.
Table 3. Performance in the AX discrimination task according to the number of speakers

\begin{tabular}{|c|c|c|c|c|c|}
\hline \multirow[b]{2}{*}{ Task } & \multirow[b]{2}{*}{$\begin{array}{l}\text { No. } \\
\text { speakers }\end{array}$} & \multicolumn{2}{|c|}{ A.T. } & \multicolumn{2}{|c|}{ F.L. } \\
\hline & & $N$ & $\%$ & $N$ & $\%$ \\
\hline \multirow[t]{2}{*}{ AX discrimination } & 1 & $58 / 60$ & 97 & $58 / 60$ & 97 \\
\hline & 2 & $56 / 60$ & 93 & $59 / 60$ & 98 \\
\hline \multirow{2}{*}{$\begin{array}{l}\text { AX discrimination } \\
\text { with auditory mask }\end{array}$} & 1 & $58 / 60$ & 97 & $58 / 60$ & 97 \\
\hline & 2 & $57 / 60$ & 95 & $57 / 60$ & 95 \\
\hline \multirow{2}{*}{$\begin{array}{l}\text { AX discrimination } \\
\text { with concurrent task }\end{array}$} & 1 & $22 / 24$ & 92 & $22 / 24$ & 92 \\
\hline & 2 & $23 / 24$ & 96 & $24 / 24$ & 100 \\
\hline
\end{tabular}

Note: Number and percentage of correct responses.

reported in Table 3. For both A.T. and F.L., there was no effect of speaker change: A.T., $\chi^{2}(1)=0.18, p=.7$, and F.L., $\chi^{2}(1)=0, p=1$. Both patients showed excellent performance, suggesting intact phonological decoding.

Processing continuous speech may be more difficult than processing words or syllables in isolation because in a sentence, each segment may be masked by the presence of adjacent segments. It is therefore important to assess the ability of the patients to perform a discrimination task in the presence of forward and backward masking noise. To test for the effect of auditory masking, we repeated the AX discrimination task with the $\mathrm{X}$ items surrounded by a $1,000-\mathrm{ms}$ mask consisting of babble noise. Stretches of babble noise were obtained by mixing 10 continuous sequences of words and pseudowords randomly selected from the set of X stimuli. The stretches were cut into chunks of $1,000 \mathrm{~ms}$ and were concatenated around each $\mathrm{X}$ stimulus after adjusting the amplitudes so that the masks and stimuli had equal root mean squares. The babble noise hence had the same average spectra and energy as the stimuli they were surrounding. This situation approximates a real-life situation where one has to monitor for a message in the middle of surrounding conversations. If the patients suffered from either auditory masking effects or auditory attention deficits, they should have difficulties performing this version of the $\mathrm{AX}$ discrimination task.

COGNITIVE NEUROPSYCHOLOGY, 2006, 23 (6) 
Patients' performance was the same with and without the babble noise mask $^{2}$ (see Table 2): A.T., $\chi^{2}(1)=0, p=1$, and F.L., $\chi^{2}(1)=0.1$, $p=.7$. As previously, we also analysed the performance according to the number of speakers within experimental trials (see Table 3). We found no effect of speaker change: A.T., $\chi^{2}(1)=0, p=1$, and F.L., $\chi^{2}(1)=0, p=1$.

In everyday life, of course, one is faced not only with concurrent noise but also with competing attentional demands. Could it be that the patients' reported difficulties in everyday-life situations are related to difficulties in performing concurrent tasks? To address this issue, we tested the patients on a dual-task paradigm: They were asked to simultaneously perform the auditory discrimination task and a letter detection task on written text. In the concurrent task, the patients were told to detect a given letter in a newspaper article and to cross out every occurrence of the given letter as fast as they could, without stopping or slowing down. As previously, in order to prevent the use of an acoustic strategy to perform the discrimination task, stimuli were produced by two different speakers on half of trials. Since the patients were no longer able to give their discrimination task response by pressing a button, they were told to give it orally (yes and no response). The experimenter monitored the patients while they did this dual task and ensured that performance letter detection task was not interrupted or slowed down. Furthermore, in order to make the task very difficult, the auditory stimuli (A and X) were, as previously, embedded in babble noise, but for 3-s instead of 1-s durations.

Results showed that even when doing a concurrent task, the patients performed very well, and performance did not differ from that of the AX discrimination task (see Table 2): A.T., $\chi^{2}(1)=0$, $p=1$, and F.L., $\chi^{2}(1)<0.1, p=.9$. Again, we analysed performance according to the number of speakers within trials (see Table 3), and there is no effect of speaker change: A.T., $\chi^{2}(1)=1.2$, $p=.3$, and F.L., $\chi^{2}(1)=0.5, p=.5$. We also checked whether the total duration of the AX discrimination task when the patients were doing the concurrent letter detection task had not increased. Indeed, if the patients were impaired by the dual task, one could expect that they would need more time to perform the discrimination task. The duration of an AX trial was about $7.5 \mathrm{~s}$ (1.5 $\mathrm{s}$ for the $\mathrm{A}$ and the $\mathrm{X}$, and $6 \mathrm{~s}$ of babble noise) and both patients spent about $8.4 \mathrm{~s}$ on each trial, a duration similar to the one observed without the concurrent task. This suggests that they were not slowed down by the letter detection task. Moreover, we calculated the number of written words scanned by each patient during the AX task. About 600 words were scanned by both patients, meaning that each word was processed in $640 \mathrm{~ms}$, showing that they were also really fast at doing the concurrent task.

Overall, these data confirm the excellent performance of the patients at decoding speech sounds, even in difficult situations.

\section{Lexical decision}

Here we tested the possibility that the patients might be impaired at lexical processing. Impaired word form selection, resulting from a deficit either in the selection process or in the longterm representation of lexical word forms, should be revealed by difficulties in discriminating known words from phonologically legal pseudowords. We used the list of 60 words and 60 pseudowords described above. Stimuli were auditorily presented, and patients had to decide whether they were real words or not by pressing a button.

The patients performed as well as control subjects on the lexical decision task (Table 2; $Z$-score $=$ $-0.84, p=.28$ ). A further analysis was performed to separate the pseudowords that were phonologically very distant from existing words from the pseudowords that were minimally distant from existing words. There were 28 pseudowords differing in one phonetic feature from a known lexical entry

\footnotetext{
${ }^{2}$ We also used babble noise mask of 3,000 ms instead of 1,000 ms in order to make the AX discrimination task more difficult but patients' performance was as good as without any mask. A.T. and F.L. discriminated correctly $96 \%$ of the items.
} 
and 32 pseudowords differing in more than one phonetic feature from a known lexical entry. In both conditions, patients performed as well as control subjects (one phonetic feature and more than one phonetic feature difference, respectively: controls, 97\% correct, A.T., $100 \%$ correct, $Z$-score $=0.7$, $p=.3$, and F.L., $93 \%$ correct, $Z$-score $=-1.2$, $p=.2 ;$ and controls, $98 \%$ correct, A.T., $100 \%$ correct, $Z$-score $=0.8, p=.3$, and F.L., $97 \%$ correct, $Z$-score $=-0.6, p=.3$ ). These results allowed us to discard the hypothesis that the patients suffer from damaged word form selection.

\section{Auditory word-picture matching}

A deficit in the retrieval of semantic information attached to lexical entries should be revealed in a task in which patients have to match a given speech input with a picture. An auditory wordpicture matching task was administered to the patients. A total of 64 pictures of common concrete nouns were selected and were visually presented immediately following the presentation of an auditory word. The word was the correct name of the picture $(N=64$; e.g., "bureau" /byro/ desk for a picture of a desk), an unrelated distractor $(N=64$; e.g., "pomme" /pom/ apple), a semantic distractor ( $N=64$; e.g., "armoire" /armwar/ wardrobe), or a phonological distractor. There were two types of phonological distractor: words $(N=64)$ and pseudowords $(N=64)$, which were equidistant from the target word (e.g., the word /bylo/ whelk and the pseudoword /byfo/). Every picture was presented once with each auditory item (the identity and the four types of distractor). The patients had to decide whether the auditory word corresponded to the picture that followed it.

Both patients performed as well as normal controls (Table 2; A.T.: Z-score $=-1, p=.3$, and F.L.: $Z$-score $=0, p=1$ ). Here again, the excellent performance of the two patients allowed us to exclude lexical retrieval deficit, as well as in the two stages preceding lexical retrieval (phonological decoding and word form selection).

\section{Discussion}

As both patients were complaining of perception difficulties, we evaluated their phonological decoding performance with an AX discrimination task. In this task, patients were presented with two bisyllabic stimuli that could either differ or not. When they differed, it was only by a single phonetic feature of one phoneme. Patients were flawless in doing the discrimination task. To ensure that the patients' difficulties in everyday life did not stem from the effect of forward or backward perceptual masking, the same task was tested with stimuli embedded in babble noise, and results confirmed the excellent performance of the patients in decoding speech sounds, even in this difficult situation. Even when increasing the difficulty of the discrimination task by asking the patients to perform a concurrent task while they were doing the discrimination task, we did not observe any drop in performance. Moreover, we should note that on half of the trials stimuli were produced by two different speakers. In this case, the discrimination task cannot be performed on the basis of acoustic information and requires the integrity of phonological decoding. Nevertheless, even in this condition, A.T. and F.L. performed accurately, and their performance was not affected by the babble noise mask or the concurrent task (see Table 3).

Word form selection was assessed with a lexical decision task. Both patients performed accurately on this task even when auditory stimulus is a pseudoword that differs from only one phoneme from a real word. Lexical retrieval was then assessed with an auditory word-picture matching task. In this task, several types of distractor were tested. In particular, each picture was tested with two phonologically related distractors (one word and one pseudoword). Again the patients' performance was flawless whatever the type of distractor.

Taken together, these results demonstrate that in both patients phonological decoding is intact. Both patients perform accurately in the discrimination task irrespective of the difficulty of the task: Neither the babble noise masking nor the concurrent task induced performance decrease. These results suggest that the patients' phonological buffer deficit does not result from phonological decoding deficit. Thus far, when using words presented in isolation, the experimental testing failed 
to reveal any deficit at any of the further stagesword form selection and lexical retrieval-that underlie the comprehension of spoken words (see Table 2). Nevertheless, despite patients' perfect word comprehension abilities they were still complaining of difficulties in processing speech sounds within sentences.

\section{SENTENCE PROCESSING}

In this third part, we investigated the patients' performance in tasks involving sentence processing. The first two tasks were constructed to evaluate the patients' ability to perform a phonological analysis of the sentence. In these two tasks the patients were required to make phonological decision about the words embedded in sentences. In the first task we asked the patients to detect word mispronunciation (words not correctly pronounced) in sentences, and in the second one we asked them to decide whether a specific word previously presented was contained or not in a sentence. We then administered two additional tasks to test the access of lexical level in sentence context: a word segmentation task and a word recognition task. We constructed these tasks such that they required resolving phonological ambiguities to be performed.

\section{Phonological analysis of the sentence}

\section{Word mispronunciation detection}

In this task, the patients had to detect a mispronounced word in sentence context. Patients were presented with a picture representing a situation (two characters, several objects, and an action) and an auditory sentence describing the picture. The auditory sentences were always the correct description of the picture, but they could contain one mispronounced word, obtained by substituting one phoneme for another. For instance "cheval" / eval/ horse became / evar/. The patients had to detect when a mispronounced word was present in the sentence. Therefore, the detection task taps the phonological decoding level of sentence processing. There were 16 correct sentences: 8 syntactically and semantically simple, for instance "le garçon attrape le cheval" (the boy grabs the horse), and 8 syntactically and semantically complex sentences, for instance "le pompier qui a des bottes noires arrose la valise" (the fireman who wears black boots waters the suitcase). This task involved both simple and complex sentences. Simple sentences were composed of five words, and the grammatical structure was subject, verb, and object. Complex sentences were composed of $9.7 \pm 0.7$ words and contained a relative clause. There were 24 incorrect sentences (12 simple and 12 complex sentences). The position of the distortion in the word (onset, midword, and offset) and the position of the mispronounced word within the sentence (onset, midsentence, and offset) were varied.

In the mispronunciation detection task, both A.T. and F.L. were impaired relative to control subjects (Table 2; A.T.: $Z$-score $=-4.1 p<.001$, and F.L.: $Z$-score $=-4.7, p<.001)$. There was no effect of the position of the distortion within the word neither with the patients, A.T., $\chi^{2}(2)=0.45, p=.9$, and F.L., $\chi^{2}(2)=3.3, p=.2$, nor with the controls $(p>.1)$. In contrast, there was a marginal effect of the position of the mispronounced word within the sentence (Table 4): A.T., $\chi^{2}(2)=4.9, \quad p=.087, \quad$ and F.L., $\quad \chi^{2}(2)=5.2$, $p=.072$. This was also observed for control subjects (Table 4$): \chi^{2}(2)=8.9, p=.01$. Both patients were impaired relative to controls in detecting mispronunciation that occurs at midsentence and at the sentence offset (respectively, A.T.: $Z$-score $=-4, \quad p<.001, \quad$ and $Z$-score $=-3.5$, $p<.001$, and F.L.: $Z$-score $=-3, p<.001$, and $Z$-score $=-5.6, p<.001)$, whereas they were not or only slightly impaired when the mispronunciation occurred at sentence onset (A.T.: Zscore $=-1.9, p=.07$, and F.L.: $Z$-score $=0.47$, $p=.36)$. We also analysed the patients' performance according to the sentence complexity. There were 20 simple and 20 complex sentences in this task. Patients' results showed that accuracy in simple and complex sentences did not differ (Table 4): A.T., $\chi^{2}(1)=0.15, p=.7$, and F.L., $\chi^{2}(1)=0$, $p=1$. This pattern was also observed in control subjects $(p>1)$. This experiment thus revealed a 
Table 4. Performance in the mispronunciation detection task according to sentence complexity and position of mispronounced word in sentence

\begin{tabular}{|c|c|c|c|c|c|c|}
\hline & & \multicolumn{2}{|c|}{ A.T. } & \multicolumn{2}{|c|}{ F.L. } & \multirow[b]{2}{*}{ Controls $^{\mathrm{b}}$} \\
\hline & & $N$ & $\%^{\mathrm{a}}$ & $N$ & $\%^{\mathrm{a}}$ & \\
\hline \multirow[t]{2}{*}{ Sentence complexity } & simple & $15 / 20$ & 75 & $17 / 20$ & 85 & $95( \pm 3.7)$ \\
\hline & complex & $15 / 20$ & 75 & $15 / 20$ & 75 & $95( \pm 6.4)$ \\
\hline \multirow{3}{*}{$\begin{array}{l}\text { Position of } \\
\text { mispronunciation in sentence }\end{array}$} & onset & $7 / 8$ & 88 & $8 / 8$ & 100 & $97( \pm 5.2)$ \\
\hline & middle & $3 / 8$ & 38 & $4 / 8$ & 50 & $87( \pm 14.4)$ \\
\hline & offset & $6 / 8$ & 75 & $5 / 8$ & 62.5 & $96( \pm 6)$ \\
\hline
\end{tabular}

${ }^{\mathrm{a}}$ Percentage of correct detections. ${ }^{\mathrm{b}}$ Standard deviations in parentheses.

significant impairment in word mispronunciation detection in the context of sentences. Patients' accuracy was lower than that of control subjects especially when the mispronunciation occurs at midsentence and at the sentence offset. The patients' deficit was not increased by sentence complexity.

\section{Word detection}

Here, we used a word detection task, which is the equivalent to the $\mathrm{AX}$ discrimination task but within a sentence context. This task taps the phonological level at the sentence-processing stage. The patients were presented with an auditory target word before hearing a sentence containing the target word, or containing a pseudoword differing from the target word for one phonetic feature of one phoneme. The patients had to indicate whether or not the target word was present in the sentence. There were 64 experimental sentences (half contained the target word, and half contained a pseudoword instead of the word). For instance, the target word was "nappe" /nap/ (tablecloth), and the sentence containing a pseudoword was "j'ai mis la nabe rustique sur la table ( $I$ put the rustic tableclosh on the table), where "nabe" /nab/ is a pseudoword. The target word was spoken by a male voice while the test word or pseudoword embedded within a sentence (mean: 8 words long) was presented in a female voice.

Results are reported in Table 2. A.T. and F.L. are impaired relative to control subjects (A.T.: $Z$-scores of $-7.7, p<.001$, and F.L.: $Z=-3.8$, $p<.001)$. These results reflect for the most part incorrect word detection responses in pseudoword sentences (19\% correct for A.T., 47\% correct for F.L.). In contrast, the detection rate when the target was present was quite high ( $84 \%$ for A.T. and $87 \%$ for F.L.). Thus, we found a clear indication of a perceptual deficit consistent with the patients' subjective reports. A minimal pair discrimination, which is very easy when the stimuli are presented in isolation, became very difficult when embedded in a sentence.

The difficulty in evaluating words in sentences may have been due to the reduced time available to the patients to make their decisions, compared to a situation of isolated word perception. Indeed, words are typically much shorter when appearing in sentence context than when they appear in isolation. If the patients' impairment in sentence context was due to a limitation of processing speed, one would expect that slowing down the speech rate of the sentences would result in a large improvement in performance. We tested the patients with the word detection task using the sentences described above, but reduced the presentation rate of the sentences by a factor of 2.2. This factor was chosen so that the average length of the test words in the sentence context would be equal to the length of the isolated test words used in the AX discrimination experiment described above. The transformation was implemented through the PSOLA algorithm available in the PRAAT speech editor (Boersma, 1992). The task and instructions were the same as before. 
These patients' results did not differ from the previous results obtained at a normal rate of speech (Table 2): A.T., $\chi^{2}(1)=0.3, p=.6$, and F.L., $\chi^{2}(1)=0.7, p=.4$, and showed the same profile of high false-alarm rates.

\section{Attentional resources}

It is widely accepted that attentional resources are more involved in sentence processing than in single-word processing. To ensure that difficulties in sentence processing were not due to an attentional processing impairment, we proposed to retest the patients with the previous word detection in sentence task but reducing the involvement of attentional resources. We defined two conditions, one requiring high attentional resources and one low attentional resources. The first one was the exact replication of the previous experiment while in the second one patients were also presented simultaneously with a written version of the sentence. The written sentence was the exact transcription of the auditory sentence, and the target word was not written but was symbolized by a blank space. It was provided ahead of time so the patients could read it carefully as long as they wanted. The auditory sentence was presented only after the patients told the experimenter that they were ready to hear it. We reasoned that the patients could anticipate when the target word would occur in the sentence and hence would be able to better focus their attentional resources. The attentional account would therefore lead one to expect better performance when an attentional cue is provided (Theodoridis \& Schoeny, 1988).

This experiment reveals no significant difference between high and low attentional resources conditions. A.T. performs $67 \%$ correct without written phrases $(N=30)$ and $68 \%$ correct with written phrases $(N=34), \chi^{2}(1)=0, p=1$, and F.L. performs $63 \%$ correct without written phrases $(N=30)$ and $70 \%$ correct with written phrases $(N=34), \chi^{2}(1)=0.12, p=.73$.

\section{Discussion}

The performance of the two patients in speechprocessing tasks involving isolated words was so good that we wondered whether they had any perceptual problem at all. Nevertheless, they insisted that they could not perceive phonological details when they occurred in the context of conversation. This incited us to use tasks similar to the one we had used before, but in a context closer to that of everyday language use. In the two tasks involving the detection of a mispronounced word or of a target word within a sentence context, both patients showed a significant impairment. The flawless performance of both patients in the AX discrimination task in which stimuli were embedded in energy-matched babble noise suggests that the patients' deficit is not due to the forward or backward masking effect of surrounding materials in the sentence.

One possible explanation for the better performance with isolated words than with sentences would be that some aspects of lexical-semantic or syntactic processing are impaired in both patients. Processing of syntactic structure does not appear to depend on phonological buffer capacities, and patients with impaired phonological buffer who can still understand syntactically complex sentences have been described (Martin, 1987, 1990). Nevertheless, it could be that syntactic or semantic processes are impaired in our patients and that they are slowing down the integration of semantic or syntactic information-for example, at the end of the sentence they would still be processing the earlier words (Romani, 1994). In this case, the phonological buffer could be useful to compensate for syntactic or semantic slow processing. Consequently, because of their difficulty with immediate syntactic or semantic processing, the patients could be more dependent on a backup phonological trace than are subjects whose syntactic and semantic processes are operating efficiently. In order to ensure that mispronunciation detection impairment was not the result of semantic or syntactic processing impairment, we compared patients' performance in simple and complex sentences in which more semantic and syntactic information had to be processed. Indeed, if the mispronunciation detection deficit was due to lexical or semantic deficit, one could expect increased difficulties in detecting mispronunciation 
occurring in complex sentences compared to simple sentences. We did not observe any advantage in processing simple sentences compared to complex sentences, suggesting that a semantic or syntactic processing impairment could not explain the patients' difficulties in processing speech sounds in sentences.

We also investigated whether the processing impairment with words in sentences could be attributed to the faster speaking rate in sentences than for a single word. When the sentences were artificially slowed down to match the average rate of words presented in isolation, their deficit remains stable. Thus any effect of the increased speaking rate to explain their word detection difficulties in sentences can be discarded.

Finally, we verified whether the patients' impairment in detecting words in sentences could be attributed to attentional deficits. Reducing the attentional load in the word detection task did not improve patients' performance, suggesting that an attentional processes impairment cannot explain the patients' perception deficits in sentences.

Overall, the presence of the sentential context makes the speech discrimination task difficult for these patients (Table 2).

\section{Accessing the lexical level}

\section{Word recognition in sentences}

As we have seen previously, patients were not able to perform tasks that involve the perception of words embedded in sentences. For instance, in sentences, they failed to detect distortions occurring in words or they failed to reject pseudowords when they were minimally different from real words. We propose that the phonological buffer supports the temporary storage of phonological input in order to resolve phonological ambiguities due, for example, to the phonological similarity between two words. Thus we predict that in sentence context the patients will not be able to differentiate a word from another if they are phonologically similar (e.g., "canal" vs. "canard", / kanal/ vs. / kanar/, canal vs. duck). To test this hypothesis, we constructed a new word detection task. In this task, the patients were presented first with an auditory sentence and second with the auditory target word. The task was to indicate by pressing a key ("Y", yes, or "N", no) on the keyboard to indicate whether or not the target was in the sentence. Two different speakers produced the sentence and the target word. In half of the trials, the target word was contained in the sentences, and in the other half it was a word differing by only one phonetic feature from the word contained in the sentence. For example the sentence was "Il y a un canal au fond du jardin" (There is a canal at the end of the garden), and the target was either "canal" (canal) or "canard" (duck). Both targets could be a possible word in the sentence, and the semantic content of the sentence was neutral, preventing the patients using semantic information to perform the task. For example, in English, it could be a sentence like "These hills are very high", and the target could be either "hills" or "heels".

This task was constructed with the 30 minimal pairs of words previously used in the AX discrimination task. Within each pair, there were four trial possibilities: The target was the same as the word in the sentence (e.g., "canal" in the sentence and as target word and "canard" in the sentence and as target word), and the target was not the same as the word in the sentence (e.g., "canal" in the sentence and "canard" as target word and vice versa). Each trial was presented twice. In total there were 240 sentences.

The results show that both patients were significantly impaired with this task compared to control subjects (Table 2; A.T.: Z-score $=-5.7$, $p<.001$, and F.L.: $Z$-score $=-3, p=.004)$.

\section{Word segmentation}

Models of word segmentation implement multiple activation of word candidates together with competition between overlapping candidates. At any point in time, all the words compatible with the currently available phonological information are activated, and overlapping candidates that share one or several phonemes inhibit one another (McClelland \& Elman, 1986; Norris, 1994). 
These processes ensure that each phoneme is ultimately assigned to one and only one word. In the TRACE model (McClelland \& Elman, 1986) successive time frames are represented by distinct units merely providing an implementation of a small phonological memory buffer for each of the successive levels of representation (features, phonemes, words). In the shortlist model, (Norris, 1994) the speech stream is segmented into phonemes, and this memorized sequence of phonemes is further parsed onto words via a competitive mechanism assembled on the fly. Therefore, one could propose that the phonological buffer is involved in the temporary storage of the speech stream that is necessary for word segmentation. In this case, we predict that both patients who present a phonological buffer deficit should be impaired in the word segmentation. In order to study lexical access in spoken sentences we used a word monitoring task (see Christophe, Peperkamp, Pallier, Block, \& Mehler, 2004). There were two conditions, one that contained a local lexical ambiguity (i.e., more than one lexical parse is temporarily available) and one that was entirely nonambiguous. For instance, the first sentence from the example below contains a local lexical ambiguity (in French).

1. Le livre racontait l'histoire d'un chat grincheux qui avait mordu un facteur (chagrin is a word)

The book told the story of a grumpy cat who had bitten a postman // (sorrow)

2. Le livre racontait l'histoire d'un chat drogué qui dormait tout le temps (chadro is a pseudoword)

The book told the story of a doped cat that slept all day long.

Up to the syllable "grin", participants cannot decide whether they heard the French word "chat" followed by a word starting with "grin", or whether they heard the French word "chagrin" at least not on the basis of the segmental information. In contrast, the second sentence contains no such lexical ambiguity, since no word in French starts with the string of phonemes "chad" $/ \int \mathrm{ad} /$.

If the phonological buffer is involved in lexical segmentation, we expect that patients should be more impaired than controls in the word monitoring in an ambiguous sentence task. We used the same material as that used by Christophe et al. (2004). A trial began with the visual presentation of the target word (e.g., "CHAT"). After that, the screen was left blank, and one sentence was played. The task was to indicate whether the target word was in the sentence. A total of 32 pairs of experimental sentences were constructed, such that within each pair, one sentence contained a local ambiguity (e.g., "chat grincheux"), while the other one was entirely unambiguous (e.g., "chat drogué"). The target was always a monosyllabic noun. Matched sentences were completely identical up to the target noun, and the sentence endings had similar syntactic and prosodic structures. In addition to the 64 experimental sentences, there were 32 distractor sentences to which participants should not respond. In all of these distractor sentences, one word had a syllable that was homophonic to the target word. In half of the sentences (16) the homophonous syllable was not word-initial (e.g., target "CHAT", distractor "un éCHAfaudage", scaffolding). In the remaining 16 distractor sentences the homophonous syllable was word-initial (as in "un CHApeau", a hat).

Results show that the patients were impaired in the word segmentation task: They failed to detect target words compared to control subjects (controls: 98\% correct; A.T.: $80 \%, Z$-score $=-8.6$, $p<.001 ;$ and F.L.: 92\%, $Z$-score $=-2.6, p=$ .01). A detailed analysis of their performance demonstrates that both patients made significantly more errors than control subjects in the ambiguous condition (control: 97\% correct; A.T.: 69\%, $Z$-score $=-7.4, \quad p<.001, \quad$ and F.L.: $88 \%$, $Z$-score $=-2.4, p=.02$ ) and that patient A.T. made also significantly more errors than did control subjects in the nonambiguous condition (controls: 99\% correct; A.T.: 91\%, $Z$-score $=-3.62$, $p<.001, \quad$ and F.L.: $97 \%, \quad Z$-score $=-0.8$, $p=.29)$. The patients' rejection results for the distractor sentences did not differ from control subjects' results: Correct response in distractor sentences was: controls, $88 \%$ correct; A.T., $84 \%$, and F.L., $84 \%, Z$-score $=-0.59, p=.33$. 


\section{Discussion}

The previous results show that A.T. and F.L. were both impaired when dealing with sentences instead of isolated words (see Table 2). Patients were impaired in the word recognition task, in which they had to decide whether a target word was present or not in a sentence that they had just heard. This task differed from the previous word detection task (section above) in two ways. First, in this task, all the items contained in the sentences were real words whereas in the previous task we used pseudowords embedded in sentences. Second, the target was given at the end of the sentence, forcing patients to attentively listen to the whole sentence. This situation, we think, is identical to everyday life situations in which we have to listen to an entire utterance to understand it and not to focus on a given specific word. The minimal pairs used in this task were those used in the AX discrimination task, in which patients' results were flawless. We can also note that in these sentences, the semantic context was neutral, and patients could have performed this task only on the basis of a phonological analysis of the sentence. Our results show that patients were impaired when processing the same words in a sentence context. Patients' results in the word segmentation task show that they are also impaired when dealing with sentences that they have to segment into distinct words especially in the ambiguous condition. In this condition, a syllable could belong to several words thereby requiring the temporary storage of phonological input to compute word segmentation and to check the process outcome.

Overall, these results show that patients were impaired in lexical access, as revealed by the segmentation task with ambiguous conditions and the word recognition task. This impairment is consistent with our hypothesis according to which the phonological buffer is involved in speech perception at a prelexical level, in the phonological analysis of the input. The idea we propose is that the phonological buffer allows the temporary storage of phonological input in order to compute word segmentation and to check phonological ambiguities. Such checking processes are perhaps not very useful when the stimuli are presented clearly and in isolation, but they help to recover the signal in the context of connected speech in everyday life situations.

\section{GENERAL DISCUSSION}

Two patients complaining of speech perception deficits in their everyday life were studied. Our claim was that their difficulties arose from verbal working-memory deficits. The pattern of deficits obtained in verbal working-memory tasks suggested that the phonological buffer was impaired while subvocal rehearsal was intact. We tested speech perception processing using tasks involving either isolated words or words embedded in sentences. Both patients showed excellent performance in all speech perception tasks involving single-item processing. However, they were impaired when processing words in sentences. Several hypotheses were proposed and tested to explain the patients' deficit in processing words in sentences. We first discarded the hypothesis of difficulty in processing speech sounds in sentences due to phonological decoding deficit, due to perceptual masking or due to slightly delayed decoding of speech sounds emphasized by high rate of speech in sentence. Then, we showed that an hypothetical lexical-semantic or syntactic impairment could not explain the difference between perception performance in isolated and sentences contexts. Finally, we ensured that the patients' impairment in sentence perception could not be attributed to an attentional deficit. Our results demonstrate that the patients are impaired in tasks requiring a phonological storage (lexical segmentation and word recognition in sentence), and we argue that the patients' deficits are due to their phonological buffer deficit. These results specify the role of the phonological buffer in sentence perception and more precisely in the phonological analysis of the speech stream (Figure 5). We propose that the phonological buffer is involved in tasks that require the storage of several syllables to be performed such as word segmentation and resolving phonological ambiguities. 


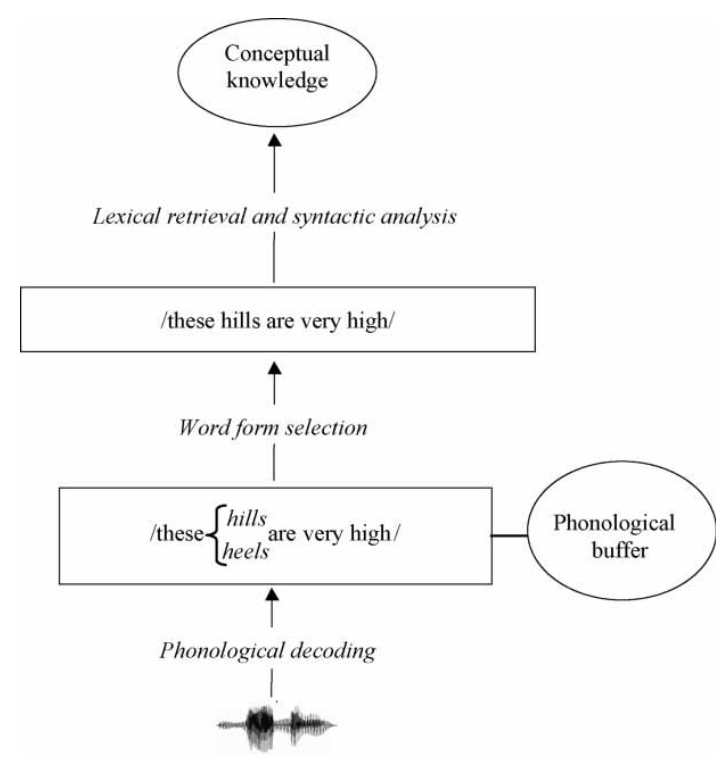

Figure 5. Model of speech perception including the phonological buffer.

Our results have several implications, both for speech perception research and for the characterization of the phonological buffer. Regarding speech perception, our two patients demonstrate a speech perception deficit that would have gone unnoticed using standard tests. A.T. and F.L. complained about difficulties in distinguishing phonologically similar words in everyday life. To our knowledge, there are no cases in the literature with a workingmemory deficit that complain of or show such difficulty. First, it should be noticed that A.T. and F.L. are both members of a society for aphasic people and are eager to better understand their deficits, and they really pay attention to their specific speech perception difficulties. Patients with severe workingmemory deficit may not complain about difficulties in perceiving small phonological contrasts because their main difficulty is to memorize information and not to deal with phonological contrasts. Second, such a deficit would not have been noticed with standard tests because most speech perception tests evaluate the processing of single words or syllables whereas the use of language in everyday life typically implies processing of connected multiword utterances. Of course, sentence comprehension tasks have been provided in many studies in patients with phonological buffer deficit, and some of them have been reported to show a good or even normal level of syntactic comprehension (Butterworth, Campbell, \& Howard, 1986; Caplan \& Waters, 1999; Hanten \& Martin, 2001, Martin, 2000; Waters et al., 1991). For instance, Vallar and Baddeley (1984b, 1987) reported that their patient, who had also a severe impairment at the phonological buffer, performed at normal level on a syntactic comprehension test that included complex sentences structures, such as reversible passive sentences. In this literature, the tasks used to assess sentence comprehension were tasks such as sentence anomaly judgement in which the patient has to decide whether the sentence is semantically or syntactically correct, or a sentence-picture matching task in which the patient has to decide whether the sentence matches with a picture. So, on the one hand, patients with phonological buffer deficit showed preserved sentence comprehension abilities, and on the other hand A.T. and F.L. showed impairment in sentence perception. Our suggestion for solving this puzzle is to note that the tasks used to assess sentence comprehension differed from those that we used in our study to evaluate sentence perception performance: The former did not tap the phonological analysis level but the ability to correctly process semantic and syntactic information (Caplan \& Waters, 1999; Martin, 2000; Van der Linden \& Poncelet, 1998). In the current study, A.T. and F.L. failed to detect a target word or a mispronounced word in a sentence. They also failed to recognize a word that differs minimally from another word, in a semantically neutral sentence. Finally, they were impaired when they had to deal with lexical ambiguities in a word segmentation task in which speech sounds could be part of one or another word. All, these tasks require the storage and the analysis of the phonological details of the sentence, and one could predict that, if tested, most of the patients with verbal working-memory deficit due to phonological buffer impairment would shown such a pattern of deficit. 
Regarding the properties of the phonological buffer, we have documented two relatively pure cases of phonological buffer impairment, in the absence of phonological decoding deficit. This confirms findings from previous studies in which patients with verbal working-memory deficits show no phonemic disorder and demonstrates that the phonological buffer can be selectively damaged without phonological decoding impairment (Basso, Spinnler, Vallar, \& Zanobio, 1982; Berndt \& Mitchum, 1990; Howard \& Nickels, 2005; Martin \& Breedin, 1992; Martin, Breedin, \& Damian, 1999a; Vallar \& Baddeley, 1984a). Further, we have suggested that A.T. and F.L. are differently impaired. In the recall task assessing the similarity effect, F.L. showed a reduced capacity of storage for both phonological similar and dissimilar sequences compared to control subjects (see Figure 3). This pattern was not observed in A.T. who showed a reduced capacity of storage only for similar sequences. This could reflect two independent aspects of the phonological buffer. One is its capacity in terms of numbers of stored elements, and another one is its resistance to interference from preceding or successive phonological segments (Nairne, 1990). One could propose that F.L. has a phonological buffer with reduced storage capacity and that A.T. has a buffer with high susceptibility to interference.

A number of researchers have explored the hypothesis that the phonological buffer is involved in acquisition of native vocabulary as well as in foreign language learning rather than in speech perception processes. The evidence supporting this possibility comes from subjects with acquired deficits as well as from developmental studies of children and from laboratory experiments on normal adults (Baddeley, 1992, 2000; Baddeley, Gathercole, \& Papagno, 1998; Baddeley \& Hitch, 2000; Baddeley, Papagno, \& Vallar, 1988; Gathercole, Service, Hitch, Adams, \& Martin, 1999; Papagno \& Vallar, 1995). The implication of these studies is that the phonological buffer is crucial to the acquisition of new phonological forms, but that semantic and lexical factors also may have a role to play (Hanten \& Martin, 2001). In this study, we showed that the role of the phonological buffer is not restricted to language acquisition. It plays a role in sentence perception and precisely at a phonological analysis level, before the access of semantic or syntactic levels. In sentence perception several syllables have to be stored in order to compute lexical segmentation and to resolve phonological ambiguities, and this is supported by the phonological buffer. In the situation of a mispronounced, misperceived, or phonologically ambiguous word, only an intact phonological buffer allows the subjects to reexamine the phonological input.

To conclude, speech comprehension involves multiple components that can be differentially affected by brain damage but that interact in normal comprehension processing (phonological, lexical, semantic, syntactic, and conceptual levels; Franklin, 1989). In models of sentences processing that assume that output from these processing levels are stored in a buffer awaiting the completion of other processes, deficits in sentence comprehension might arise not only from processing deficits, but also because of buffer deficits. Thus singleword processing may be intact, while the capacity of the buffer may be damaged, eliciting sentence comprehension deficits. A.T. and F.L. show (a) phonological buffer impairment and (b) phonological processing deficit restricted to sentences, with an intact speech decoding sysem. We argue that the dissociation between performance for isolated word and for words embedded in sentences is due to the phonological buffer impairment. The phonological buffer maintains the activation of the phonological input and allows phonological rechecking if required, and it is therefore involved in performing lexical segmentation and in resolving phonological ambiguities in sentences. Further work is needed to define and fully integrate the several storage components (phonological, lexical-semantic, syntactic, etc.) that participate in sentence comprehension processing.

Manuscript received 7 June 2004 Revised manuscript received 14 June 2005 Revised manuscript accepted 7 February 2006 First published online 22 June 2006 


\section{REFERENCES}

Baddeley, A. (1986). Working memory. New York: Oxford University Press.

Baddeley, A. (1992). Working memory. Science, 255, 556-559.

Baddeley, A. (2000). The episodic buffer: A new component of working memory? Trends in Cognitive Sciences, 4, 417-423.

Baddeley, A., Chincotta, D., Stafford, L., \& Turk, D. (2002). Is the word length effect in STM entirely attributable to output delay? Evidence from serial recognition. Quarterly Journal of Experimental Psychology, 55A, 353-369.

Baddeley, A., Gathercole, S., \& Papagno, C. (1998). The phonological loop as a language learning device. Psychological Review, 105, 158-173.

Baddeley, A., \& Hitch, G. (2000). Development of working memory. Should the Pascual-Leone and the Baddeley and Hitch models be merged? Journal of Experimental Child Psychology, 77, 128-137.

Baddeley, A., Lewis, V., \& Vallar, G. (1984). Exploring the articulatory loop. Quarterly Journal of Experimental Psychology, 36A, 233-252.

Baddeley, A., Papagno, C., \& Vallar, G. (1988). When long term learning depends on short term storage. Journal of Memory and Language, 27, 586-596.

Basso, A., Spinnler, H., Vallar, G., \& Zanobio, M. (1982). Left hemisphere damage and selective impairment of auditory verbal short-term memory. A case study. Neuropsychologia, 20, 263-274.

Belleville, S., Peretz, I., \& Arguin, M. (1992). Contribution of articulatory rehearsal to short-term memory: Evidence from a case of selective disruption. Brain and Language, 43, 713-746.

Berndt, R. S., \& Mitchum, C. S. (1990). Auditory and lexical information sources in immediate recall: Evidence from a patient with deficit to the phonological short-term buffer. In G. Vallar \& T. Shallice (Eds), Neuropsychological impairments of short-term memory. Cambridge, UK: Cambridge University Press.

Boersma, P., \& Weenink, D. (1992-2001). Praat, A system for doing phonetics by computer. Retrieved Nov. 6, 2000, from www.praat.org

Brown, G. D. A., \& Hulme, C. (1995). Modeling item length effects in memory span: No rehearsal needed? Journal of Memory and Language, 34, 594-621.

Butterworth, B., Campbell, R., \& Howard, D. (1986). The uses of short-term memory: A case study. The Quarterly Journal of Experimental Psychology, 38A, 705-737.

\section{8}

COGNITIVE NEUROPSYCHOLOGY, 2006, 23 (6)
Caplan, D., \& Waters, G. (1990). Short-term memory and language comprehension, a critical review of the neuropsychological literature. In G. Vallar \& T. Shallice (Eds.), Neuropsychological impairments of short-term memory. Cambridge, UK: Cambridge University Press.

Caplan, D., \& Waters, G. (1999). Verbal working memory and sentence comprehension. Behavioral and Brain Sciences, 22, 77-126.

Caramazza, A., Berndt, R. S., Basili, A. G., \& Koller, J. J. (1981). Syntactic processing deficits in aphasia. Cortex, 17, 333-348.

Christophe, A., Peperkamp, S., Pallier, C., Block, E., \& Mehler, J. (2004). Phonological phrase boundaries constrain lexical access: I. Adult data. Journal of Memory and Language, 51, 523-547.

Conrad, R. (1963). Acoustic confusions and memory span for words. Nature, 197, 1029-1030.

Conrad, R. (1964). Acoustic confusion and immediate memory. British Journal of Psychology, 55, 75-84.

Content, A., Mousty, P., \& Radeau, M. (1990). Brulex, Une base de données lexicales informatisées pour le français écrit et parlé. L'Année Psychologique, 90, 551-566.

Cowan, N. (2001). The magical number 4 in short-term memory: A reconsideration of mental storage capacity. Behavioral and Brain Sciences, 24, 87-114.

Cowan, N., Baddeley, A. D., Elliott, E. M., \& Norris, J. (2003). List composition and the word length effect in immediate recall: A comparison of localist and globalist assumptions. Psychonomic Bulletin $\mathcal{\sigma}^{\circ}$ Review, 10, 74-79.

Crowder, R. G. (1978). Memory for phonologically uniform lists. Journal of Verbal Learning and Verbal Behavior, 17, 73-89.

Franklin, S. (1989). Dissociations in auditory word comprehension: Evidence from nine fluent aphasic patients. Aphasiology, 3, 189-207.

Gaskell, M., \& Marslen-Wilson, W. (1996). Phonological variation and inference in lexical access. Journal of Experimental Psychology: Human Perception and Performance, 22, 144-158.

Gaskell, M., \& Marslen-Wilson, W. (1998). Mechanisms of phonological inference in speech perception. Journal of Experimental Psychology: Human Perception and Performance, 24, 380-396.

Gathercole, S., Service, E., Hitch, G., Adams, A., \& Martin, A. (1999). Phonological short-term memory and vocabulary development: Further evidence on the nature of the relationship. Applied Cognitive Psychology, 13, 65-77. 
Goodglass, H., \& Kaplan, E. (1983). Boston Diagnostic Aphasia Examination. Philadelphia: Lea \& Febiger.

Hanten, G., \& Martin, R. C. (2001). A developmental phonological short-term memory deficit: A case study. Brain and Cognition, 45, 164-188.

Howard, D., \& Nickels, L. (2005). Separating input and output phonology: Semantic, phonological, and orthographic effects in short-term memory impairment. Cognitive Neuropsychology, 22, 42-77.

Hulme, C., Maugham, S., \& Brown, G. D. A. (1991). Memory for familiar and unfamiliar words: Evidence for a long-term memory contribution to short-term memory span. Journal of Memory and Language, 30, 685-701.

Hulme, C., Surprenant, A. M., Bireta, T. J., Stuart, G., \& Neath, I. (2004). Abolishing the word-length effect. Journal of Experimental Psychology: Learning, Memory, and Cognition, 30, 98-106.

Jacquemot, C., Dupoux, E., Pallier, C., \& BachoudLévi, A.-C. (2002). Comprehending spoken words without hearing phonemes: A case study. Cortex, 38, 869-873.

Jones, D. M., Macken, W. J., \& Nicholls, A. P. (2004). The phonological store of working memory: Is it phonological and is it a store? Journal of Experimental Psychology: Learning, Memory, and Cognition, 30, 656-674.

Martin, R. C. (1987). Articulatory and phonological deficits in short-term memory and their relation to syntactic processing. Brain and Language, 32, 159-192.

Martin, R. C. (1990). Neuropsychological evidence on the role of short-term memory in sentence processing. In G. Vallar \& T. Shallice (Eds.), Neuropsychological impairments of short-term memory. Cambridge, UK: Cambridge University Press.

Martin, R. C. (1993). Short-term memory and sentence processing: Evidence from neuropsychology. Memory and Cognition, 21, 176-183.

Martin, R. C. (2000). Sentence comprehension deficits. In B. Rapp (Ed.), Handbook of cognitive neuropsychology. Philadelphia: Psychology Press.

Martin, R. C., \& Breedin, S. D. (1992). Dissociations between speech perception and phonological shortterm memory deficits. Cognitive Neuropsychology, 9, 509-534.

Martin, R. C., Breedin, S. D., \& Damian, M. F. (1999a). The relation of phoneme discrimination, lexical access, and short-term memory: A case study and interactive activation account. Brain and Language, 70, 437-482.
Martin, R. C., \& Feher, E. (1990). The consequences of reduced memory span for the comprehension of semantic versus syntactic information. Brain and Language, 38, 1-20.

Martin, R. C., \& He, T. (2004). Semantic short-term memory and its role in sentence processing: A replication. Brain and Language, 89, 76-82.

Martin, R. C., Lesch, M. F., \& Bartha, M. C. (1999b). Independence of input and output phonology in word processing and short-term memory. Journal of Memory and Language, 41, 3-29

Martin, R. C., \& Romani, C. (1994). Verbal working memory and sentence comprehension: A multiple components view. Neuropsychology, 8, 506-523.

Martin, N., \& Saffran, E. (1997). Language and auditory-verbal short-term memory impairments: Evidence for common underlying processes. Cognitive Neuropsychology, 14, 641-682.

Martin, R. C., Shelton, J., \& Yaffee, L. (1994). Language processing and working memory: Neuropsychological evidence for separate phonological and semantic capacities. Journal of Memory and Language, 33, 83-111.

Mazaux, J. M., \& Orgogozo, J. M. (1982). Echelle d'evaluation de l'aphasie: Adaptation française $d u$ Boston Diagnostic Aphasia Examination. Issy-lesMoulineaux, France: Editions Scientifiques et Psychologiques.

McClelland, J. L., \& Elman, J. L. (1986). The TRACE model of speech perception. Cognitive Psychology, 18, $1-86$.

Morton, J., Crowder, R. G., \& Prussin, H. A. (1971). Experiments with the stimulus suffix effect. Journal Experimental Psychology, 91, 169-190.

Morton, J., Marcus, S., \& Ottley, P. (1981). The acoustic correlates of speech like: A use of the suffix effect. Journal of Experimental Psychology, 110, 568-591.

Mueller, S. T., Seymour, T. L., Kieras, D. E., \& Meyer, D. E. (2003). Theoretical implications of articulatory duration, phonological similarity, and phonological complexity in verbal working memory. Journal of Experimental Psychology: Learning, Memory, and Cognition, 29, 1353-1380.

Nairne, J. S. (1990). A feature model of immediate memory. Memory \& Cognition, 18, 251-269.

Nairne, J. S. (2002). Remembering over the short-term: The case against the standard model. Annual Review of Psychology, 53, 53-81. 
Norris, D. (1994). Shortlist: A connectionist model of continuous speech recognition. Cognition, 52, 189-234.

Papagno, C., \& Vallar, G. (1995). Short-term memory and vocabulary learning in polyglots. Quarterly Journal of Experimental Psychology, 48A, 98-107.

Peperkamp, S., \& Dupoux, E. (2002). A typological study of stress "deafness". In C. Gussenhoven \& N. Warner (Eds.), Laboratory phonology 7. Berlin, Germany: Mouton de Gruyter.

Romani, C. (1994). The role of phonological short-term memory in syntactic parsing: A case study. Language and Cognitive Processes, 9, 29-67.

Romani, C., McAlpine, S., Olson, A., Tsouknida, E., \& Martin, R. C. (2005). Length, lexicality, and articulatory suppression in immediate recall: Evidence against the articulatory loop. Journal of Memory and Language, 52, 398-415.

Saffran, E., \& Marin, O. (1975). Immediate memory for word lists and sentences in a patient with deficient auditory short-term memory. Brain and Language, 2, 420-433.

Saffran, E., \& Martin, N. (1990). Short-term memory impairment and sentence processing: A case study. In G. Vallar \& T. Shallice (Eds.), Neuropsychological impairments of short-term memory. Cambridge, UK: Cambridge University Press.
Theodoridis, G. C., \& Schoeny, Z. G. (1988). Comparison of various modes of presenting sentence materials in tests of speech perception in noise. Journal of Acoustic Society of America, 84, 2270-2272.

Vallar, G., \& Baddeley, A. (1984a). Fractionation of working memory: Neuropsychological evidence for a phonological short-term buffer. Journal of Verbal Learning and Verbal Behavior, 23, 151-161.

Vallar, G., \& Baddeley, A. D. (1984b). Phonological short-term store: Phonological processing and sentence comprehension. Cognitive Neuropsychology, 1, 121-141.

Vallar, G., \& Baddeley, A. D. (1987). Phonological short-term store and sentence processing. Cognitive Neuropsychology, 4, 417-438.

Vallar, G., \& Papagno, C. (1986). Phonological shortterm buffer and the nature of the recency effect: Evidence from neuropsychology. Brain and Cognition, 5, 428-442.

Van der Linden, M., \& Poncelet, M. (1998). The role of working memory in language and communication disorders. In B. Stemmer \& H. A. Whitaker (Eds.), Handbook of neurolinguistics. San Diego, CA: Academic Press.

Waters, G., Caplan, D., \& Hildebrandt, N. (1991). On the structure of verbal short-term memory and its functional role in sentence comprehension: Evidence from neuropsychology. Cognitive Neuropsychology, 8, 81-126. 


\section{APPENDIX A}

Performance of patient A.T. on a French version of the Boston Diagnostic Aphasia Examination (Goodglass E Kaplan, 1983; Mazaux EO Orgogozo, 1982)

\begin{tabular}{|c|c|c|c|}
\hline & Subtests & Scores & $\begin{array}{c}\text { Normal } \\
\text { range } \\
\text { limits }\end{array}$ \\
\hline \multirow{4}{*}{$\begin{array}{l}\text { Auditory } \\
\text { comprehension }\end{array}$} & Word discrimination & $70 / 72$ & $N>55$ \\
\hline & $\begin{array}{l}\text { Body-part } \\
\quad \text { identification }\end{array}$ & $16 / 20$ & $N>15$ \\
\hline & Command & $10 / 15$ & $N>10$ \\
\hline & Logical reasoning & $9 / 12$ & $N>5$ \\
\hline \multirow[t]{2}{*}{ Fluency } & Phrase length & $7 / 7$ & $N>5$ \\
\hline & Verbal agility & $11 / 14$ & $N>6$ \\
\hline \multirow{2}{*}{$\begin{array}{l}\text { Automatic } \\
\text { speech }\end{array}$} & Automatized sequences & $9 / 9$ & $N>5$ \\
\hline & Reciting & $2 / 2$ & $N>1$ \\
\hline \multirow[t]{3}{*}{ Repetition } & Repetition of words & $8 / 10$ & $N>7$ \\
\hline & Concrete sentences & $6 / 8$ & $N>5$ \\
\hline & Abstract sentences & $5 / 8$ & $N>2$ \\
\hline \multirow[t]{2}{*}{ Reading aloud } & Words & $30 / 30$ & $N>19$ \\
\hline & Sentences & $10 / 10$ & $N>5$ \\
\hline \multirow[t]{4}{*}{ Naming } & Responsive naming & $24 / 30$ & $N>22$ \\
\hline & $\begin{array}{l}\text { Confrontation } \\
\text { naming }\end{array}$ & $100 / 105$ & $N>70$ \\
\hline & Animal naming & $20 / 23$ & $N>7$ \\
\hline & Body-part naming & $28 / 30$ & $N>19$ \\
\hline \multirow[t]{2}{*}{ Paraphasia } & $\begin{array}{l}\text { Phonological Eळ } \\
\text { morphological }\end{array}$ & $4 / 16$ & $N<5$ \\
\hline & Semantic & $5 / 24$ & $N<7$ \\
\hline \multirow{5}{*}{$\begin{array}{l}\text { Written } \\
\text { comprehension }\end{array}$} & Literal discrimination & $10 / 10$ & $N>7$ \\
\hline & $\begin{array}{c}\text { Auditory-written- } \\
\text { word matching }\end{array}$ & $8 / 8$ & $N>6$ \\
\hline & Spelled words & $5 / 8$ & $N>3$ \\
\hline & $\begin{array}{l}\text { Picture-written-word } \\
\quad \text { matching }\end{array}$ & $10 / 10$ & $N>7$ \\
\hline & Text reading & $8 / 10$ & $N>5$ \\
\hline \multirow[t]{5}{*}{ Writing } & Mechanics & $3 / 3$ & $N>2$ \\
\hline & Dictation & $14 / 15$ & $N>10$ \\
\hline & Written denomination & $10 / 10$ & $N>5$ \\
\hline & Graphical evocation & $9 / 10$ & $N>4$ \\
\hline & Sentence dictation & $12 / 12$ & $N>5$ \\
\hline
\end{tabular}

\section{APPENDIX B}

Performance of patient F.L. on a French version of the Boston Diagnostic Aphasia Examination (Goodglass E Kaplan, 1983; Mazaux E' Orgogozo, 1982)

\begin{tabular}{|c|c|c|c|}
\hline & Subtests & Scores & $\begin{array}{c}\text { Normal } \\
\text { range } \\
\text { limits }\end{array}$ \\
\hline \multirow{4}{*}{$\begin{array}{l}\text { Auditory } \\
\text { comprehension }\end{array}$} & Word discrimination & $71 / 72$ & $N>55$ \\
\hline & $\begin{array}{l}\text { Body-part } \\
\quad \text { identification }\end{array}$ & $18 / 20$ & $N>15$ \\
\hline & Command & $13 / 15$ & $N>10$ \\
\hline & Logical reasoning & $9 / 12$ & $N>5$ \\
\hline \multirow[t]{2}{*}{ Fluency } & Phrase length & $7 / 7$ & $N>5$ \\
\hline & Verbal agility & $12 / 14$ & $N>6$ \\
\hline \multirow{2}{*}{$\begin{array}{l}\text { Automatic } \\
\text { speech }\end{array}$} & Automatized sequences & $9 / 9$ & $N>5$ \\
\hline & Reciting & $1 / 2$ & $N>1$ \\
\hline \multirow[t]{3}{*}{ Repetition } & Repetition of words & $8 / 10$ & $N>7$ \\
\hline & Concrete sentences & $4 / 8$ & $N>5$ \\
\hline & Abstract sentences & $3 / 8$ & $N>2$ \\
\hline \multirow[t]{2}{*}{ Reading aloud } & Words & $30 / 30$ & $N>19$ \\
\hline & Sentences & $10 / 10$ & $N>5$ \\
\hline \multirow[t]{4}{*}{ Naming } & Responsive naming & $30 / 30$ & $N>22$ \\
\hline & $\begin{array}{l}\text { Confrontation } \\
\text { naming }\end{array}$ & $95 / 105$ & $N>70$ \\
\hline & Animal naming & $15 / 23$ & $N>7$ \\
\hline & Body-part naming & $29 / 30$ & $N>19$ \\
\hline \multirow[t]{2}{*}{ Paraphasia } & $\begin{array}{l}\text { Phonological Eळ } \\
\text { morphological }\end{array}$ & $6 / 16$ & $N<5$ \\
\hline & Semantic & $6 / 24$ & $N<7$ \\
\hline \multirow{5}{*}{$\begin{array}{l}\text { Written } \\
\text { comprehension }\end{array}$} & Literal discrimination & $10 / 10$ & $N>7$ \\
\hline & $\begin{array}{l}\text { Auditory-written- } \\
\text { word matching }\end{array}$ & $8 / 8$ & $N>6$ \\
\hline & Spelled words & $4 / 8$ & $N>3$ \\
\hline & $\begin{array}{l}\text { Picture-written- } \\
\text { word matching }\end{array}$ & $10 / 10$ & $N>7$ \\
\hline & Text reading & $8 / 10$ & $N>5$ \\
\hline \multirow[t]{5}{*}{ Writing } & Mechanics & $3 / 3$ & $N>2$ \\
\hline & Dictation & $14 / 15$ & $N>10$ \\
\hline & Written denomination & $10 / 10$ & $N>5$ \\
\hline & Graphical evocation & $9 / 10$ & $N>4$ \\
\hline & Sentence dictation & $7 / 12$ & $N>5$ \\
\hline
\end{tabular}

